\title{
Hypercalcemia due to Milk-Alkali Syndrome and Fracture-Induced Immobilization in an Adolescent Boy with Hypoparathyroidism
}

\author{
Rohan K. Henrya Rachel I. Gafni ${ }^{b}$ \\ a Section of Endocrinology, Metabolism and Diabetes, Nationwide Children's Hospital, Columbus, Ohio, and \\ ${ }^{b}$ Craniofacial and Skeletal Diseases Branch, National Institute of Dental and Craniofacial Research (NIDCR), National \\ Institute of Health (NIH), Bethesda, Md., USA
}

\section{Established Facts}

- Immobilization hypercalcemia is more common in adolescent boys.

- Chronic high doses of calcium carbonate can result in the milk-alkali syndrome.

\section{Novel Insights}

- Hypoparathyroidism is not protective for immobilization hypercalcemia.

- Decreased bone formation in growing adolescents with hypoparathyroidism who are immobilized may contribute to hypercalcemia and milk-alkali syndrome.

\section{Key Words}

Parathyroid hormone - Fracture - Bone formation .

Hypercalcemia $\cdot$ Immobilization

\begin{abstract}
Background: Hypercalcemia of immobilization, while rare, may occur in adolescent boys after fracture. Although not fully understood, the mechanism appears to be related to bone turnover uncoupling, in part mediated by upregulation of RANKL. Animal studies suggest that parathyroidectomy suppresses RANKL-stimulated osteoclastogenesis in immobilized bone. Thus, immobilization-induced hypercalcemia should be uncommon in patients with hypoparathy-
\end{abstract}

\section{KARGER}

(c) 2016 S. Karger AG, Basel

E-Mail karger@karger.com

www.karger.com/hrp roidism. Methods/Results: We present a 15-year-old boy with well-controlled hypoparathyroidism who developed hypercalcemia and milk-alkali syndrome 5 weeks after sustaining a severe tibia/fibula fracture requiring bedrest. Milkalkali syndrome (hypercalcemia, alkalosis, and renal insufficiency) results from chronic excessive ingestion of calcium and absorbable alkali. Prior to fracture, our patient had not experienced hypercalcemia despite high doses of supplements, necessary during puberty. Supplements were discontinued and his biochemistries normalized with saline diuresis and a dose of pamidronate. Alkaline phosphatase, which was low at presentation, returned to normal 5 weeks later with remobilization. Conclusions: Fracture and immobilization caused acute suppression of bone formation with per-
Rohan K. Henry, MD, MS

Nationwide Children's Hospital

555 South 18th Street

Columbus, OH 43205 (USA)

E-Mail rohan.henry@ nationwidechildrens.org 
sistent bone resorption in this rapidly growing adolescent; continuation of carbonate-containing calcium supplements resulted in the milk-alkali syndrome. Therefore, close monitoring of serum calcium with adjustments in supplementation are indicated in immobilized patients with hypoparathyroidism.

(c) 2016 S. Karger AG, Basel

\section{Introduction}

Immobilization-induced hypercalcemia is most commonly seen in individuals experiencing rapid bone turnover, such as patients with spinal cord injuries or Paget's disease. While rare in healthy individuals, it may occur in adolescent males undergoing a pubertal growth spurt, particularly after a fracture $[1,2]$. While the exact mechanism is unknown, it is thought to be due to an uncoupling of bone turnover with an increase in bone resorption coupled with a decrease in bone formation. The role of parathyroid hormone $(\mathrm{PTH})$ in immobilization is unclear; however, parathyroidectomy prior to fracture/immobilization appears to blunt bone resorption in animals [3-5]. As such, one may expect patients with hypoparathyroidism to be somewhat protected from immobilization-induced hypercalcemia.

Milk-alkali syndrome is hypercalcemia associated with metabolic alkalosis and renal insufficiency due to excessive ingestion of calcium and absorbable alkali. Calcium salts and active vitamin D (e.g. calcitriol) are the mainstay of therapy for patients with hypoparathyroidism, with the goal of keeping the blood calcium levels near or just slightly below the lower limit of normal while avoiding hypercalciuria. Medication doses are highly individualized, with some patients requiring several grams of elemental calcium daily, particularly during periods of rapid growth in adolescence. We present here an adolescent boy with hypoparathyroidism who developed the milk-alkali syndrome following fracture with immobilization.

\section{Case Presentation}

The patient is a 15-year-old white male with autoimmune polyendocrinopathy-candidiasis-ectodermal dystrophy syndrome (APECED), manifesting as hypoparathyroidism and chronic thrush without adrenal insufficiency. Growth and development were normal (50-75\% for height and weight, Tanner IV puberty) and he was otherwise healthy. His serum calcium levels were wellmaintained at $7.6-8.2 \mathrm{mg} / \mathrm{dl}$ on calcitriol $0.75 \mu \mathrm{g} /$ day and calcium carbonate (3 g elemental calcium/day in divided doses). While playing sports, he sustained a severe traumatic tibia/fibula fracture; blood work done on the day of fracture showed a serum calcium of $7.6 \mathrm{mg} / \mathrm{dl}$, serum creatinine of $0.88 \mathrm{mg} / \mathrm{dl}$, and bicarbonate of 26 $\mathrm{mmol} / \mathrm{l}$. No changes were made to his medication regimen. Fracture treatment required casting and significant immobilization, including bedrest for 2 weeks and very limited activity thereafter. Three to four weeks after the fracture, the patient developed fatigue, headache, vomiting, abdominal pains, polyuria and polydipsia. Five weeks after the fracture, he presented to an emergency department and was found to be hypertensive with a blood pressure of 140/65 and markedly hypercalcemic with a serum calcium of $13.5 \mathrm{mg} / \mathrm{dl}$ and phosphate of $3.9 \mathrm{mg} / \mathrm{dl}$ (fig. 1a).

Additional studies revealed metabolic alkalosis and acute renal insufficiency with serum bicarbonate of $34 \mathrm{mmol} / \mathrm{l}$ (normal 22-29 $\mathrm{mmol} / \mathrm{l}$ ) and serum creatinine 1.9 (normal 0.6-1.2 mg/dl). Potassium was normal at $3.9 \mathrm{mmol} / \mathrm{l}$. An EKG showed a normal QT interval with sloping $\mathrm{T}$-waves. Serum alkaline phosphate level was $132 \mathrm{U} / \mathrm{l}$, in the low end of the normal range for age (fig. 1b). On previous measure, 8 months prior, the alkaline phosphatase was 202 U/l. Serum N-telopeptide at the time of fracture was reported as $>40 \mathrm{nmol} / \mathrm{l} \mathrm{BCE} \mathrm{(enzyme} \mathrm{immunoassay,} \mathrm{Quest} \mathrm{Specialty} \mathrm{Labo-}$ ratories, Santa Monica, Calif., USA). While the normal range for men $\geq 19$ years is $5.4-24.2 \mathrm{nmol} / \mathrm{l} \mathrm{BCE}$, pediatric normal ranges were not available; thus, it is not known if bone resorption was elevated or appropriate for a pubertal boy. Evaluation for malignancy was unremarkable and PTH-related peptide (PTHrP) was undetectable. $25-\mathrm{OH}$ vitamin $\mathrm{D}$ was normal at $56 \mathrm{ng} / \mathrm{ml}$ and, although the patient was taking calcitriol, the $1,25-\mathrm{OH}_{2}$ vitamin $\mathrm{D}$ level was low at $14.5 \mathrm{pg} / \mathrm{ml}(25.1-66.1 \mathrm{pg} / \mathrm{ml})$. Intact PTH was undetectable, as expected.

Treatment for hypercalcemia included discontinuation of calcium carbonate and calcitriol, low calcium diet, aggressive saline diuresis, and intermittent furosemide. On hospital day 3, pamidronate $(0.5 \mathrm{mg} / \mathrm{kg})$ was administered with a decrease in serum calcium from 12.5 to $11.2 \mathrm{mg} / \mathrm{dl}$ within $6 \mathrm{~h}$. Serum phosphate levels fluctuated but stayed within the normal range, except for an acute drop 1 day after the pamidronate. The patient was discharged on day 4 with continued outpatient monitoring (fig. 1a).

Calcium supplements (as calcium citrate) and calcitriol were restarted 2 days after discharge when his serum calcium was 8.8 $\mathrm{mg} / \mathrm{dl}$ and was adjusted as needed to maintain calcium levels within his target range of 7.6-8.2 mg/dl. Serum creatinine and bicarbonate gradually decreased and were back to baseline 6 days after discharge. With increasing mobilization after discharge, his alkaline phosphatase level rose steadily, returning to levels consistent with a growing adolescent boy (fig. 1b). A renal ultrasound done 5 weeks after discharge showed stable nephrocalcinosis, unchanged from previous studies.

\section{Discussion}

In this patient with hypoparathyroidism, sudden immobilization following a severe fracture was associated with hypercalcemia. The mechanism of immobilizationinduced hypercalcemia is not entirely understood, although it is thought to be due to decreased mechanical 


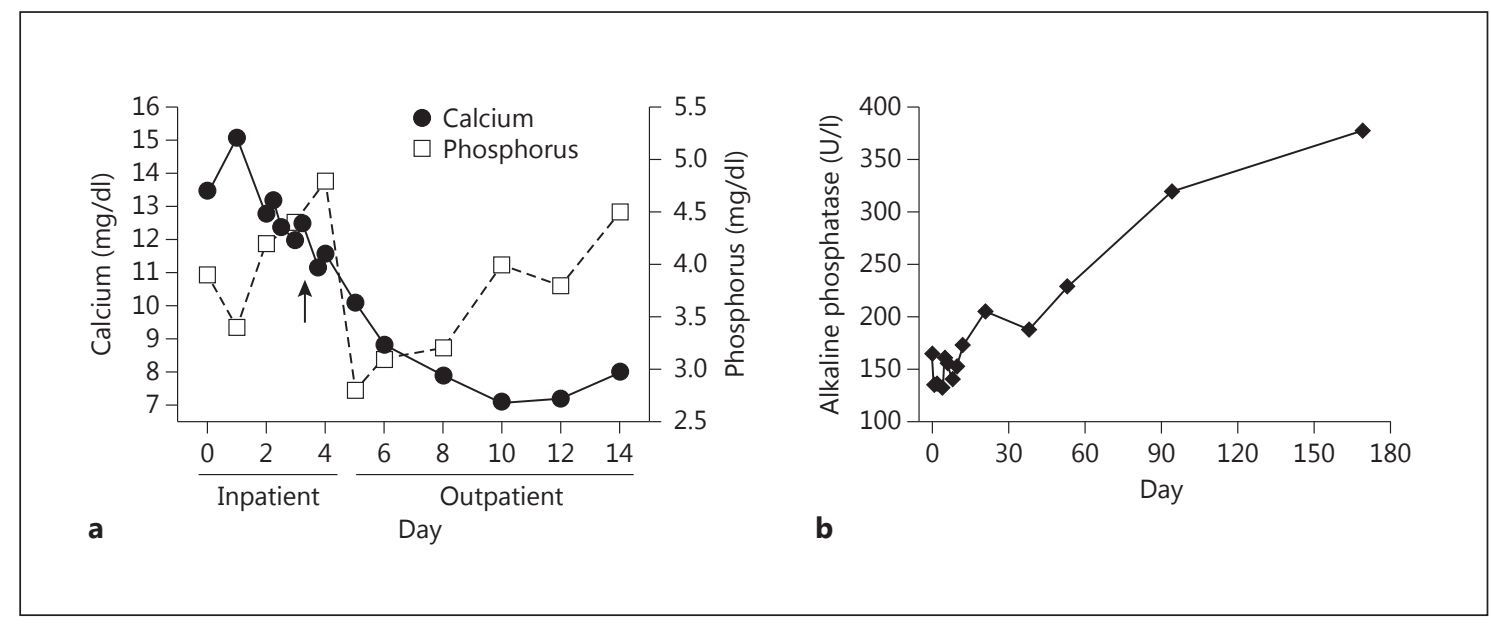

Fig. 1. a Blood calcium and phosphorus levels during and after hospitalization. Calcium target range for hypoparathyroidism: 7.6-8.2 mg/dl. Phosphorus normal range for 15-year-old boys: 3-6 mg/dl. Arrow denotes day of pamidronate infusion. b Blood alkaline phosphatase levels. Normal range for 15-year-old boys: 100-390 IU/1.

stimulation of osteoblast-driven bone formation coupled with unchecked osteoclast-mediated bone resorption [69]. Hypercalciuria develops, ultimately leading to hypercalcemia when the renal threshold for calcium is exceeded and/or renal insufficiency develops. It is often seen in highly active patients who are abruptly immobilized [10], such as spinal cord injured patients [6] and, as in our patient, rapidly growing adolescent boys [1]. That bone formation was decreased in this patient is supported by the relatively low alkaline phosphatase seen at presentation, which later increased into the normal pubertal range with remobilization.

The role of PTH in immobilization-induced hypercalcemia is unclear, as PTH levels have been reported as elevated, normal, or suppressed $[6,8,11]$. In the earliest case, Albright et al. [1] reported an immobilized 14-yearold boy who had clinically apparent hyperparathyroidism that recovered with remobilization. Experimental models have suggested that PTH stimulates receptor activator of NF- $\kappa B$ ligand (RANKL) expression in immobilized bone, promoting osteoclastogenesis and increased bone resorption [3]. When animals were parathyroidectomized prior to immobilization, RANKL expression and osteoclast number did not increase and bone loss was prevented, suggesting that PTH may be, in part, mediating bone resorption during immobilization. Thus, one might expect that hypoparathyroidism would have been protective and prevented the hypercalcemia in our patient. However, in contrast to our patient, the mice did not receive calcium or calcitriol supplementation and were very hypocalce-

Immobilization Hypercalcemia and MilkAlkali Syndrome in Hypoparathyroidism mic during immobilization, suggesting that the hypercalcemia seen in our patient was due to decreased bone formation with continued supplementation during immobilization, rather than increased bone resorption. While adults with hypoparathyroidism typically have low/lownormal bone turnover leading to increased bone density [12], in our NIH cohort of over 30 children with uncomplicated hypoparathyroidism, bone density is not increased (unpublished data). Thus, despite the absence of PTH, bone remodeling appears to proceed normally in growing children.

High doses of calcium and active vitamin D are often required to achieve serum calcium levels in the lower end of normal range in patients with hypoparathyroidism, particularly during periods of rapid growth. In this case, the hypercalcemia with presumed decreased bone formation was exacerbated by the continuation of large doses of calcium carbonate and calcitriol during the period of immobilization, leading to the milk-alkali syndrome. The origin of the milk-alkali syndrome dates back to the early 20 th century, when patients were treated for ulcer disease with the 'Sippy Method,' a complex regimen involving large amounts of milk, cream, calcinated magnesium, sodium bicarbonate, and bismuth subcarbonate [13]. Toxicity manifests as headache, nausea, vomiting, dizziness, weakness, musculoskeletal pain, polyuria and dehydration with hypercalcemia, hyperphosphatemia, metabolic alkalosis, and renal insufficiency [14]. With the development of acid blockers, the syndrome is now more commonly associated with the use of calcium salts for the 
treatment of osteoporosis in postmenopausal women. As this newer form is not associated with excess dairy intake and hyperphosphatemia, the term calcium-alkali syndrome has emerged [15]. A vicious cycle ensues whereby hypercalcemia leads to decreased glomerular filtration rate, which in turn reduces calcium filtration. Alkalosis increases renal reabsorption of calcium through numerous mechanisms, including likely stimulation of the epithelial calcium channel TRPV5 (transient receptor potential 5) [16], while also possibly increasing the sensitivity of the calcium-sensing receptor (CaSR) [14], which promotes hypercalciuria and worsens the volume depletion. Simultaneously, dehydration may stimulate angiotensin 2 production, which can cause hypokalemia and increase renal bicarbonate reabsorption, further exacerbating the alkalosis [17]. In euparathyroid individuals, hypercalcemia and CaSR activation suppresses PTH secretion, which decreases calcium efflux from bone and promotes renal calcium excretion; thus, one might expect a patient with hypoparathyroidism to be somewhat protected from developing the milk-alkali syndrome.

Hypercalcemia due to the milk-alkali syndrome has been previously reported in hypoparathyroid patients who have been overtreated with supplements or have other complex medical problems associated with renal insufficiency [18-21]. This is in contrast to our patient, who was otherwise healthy and well managed with a calcium level of $7.6 \mathrm{mg} / \mathrm{dl}$ prior to sustaining his fracture. The abrupt immobilization caused a change in bone balance, with decreased bone formation occurring in the presence of ongoing bone resorption. Coupled with inadvertent over-replacement with calcium carbonate and calcitriol, the milk-alkali syndrome developed. It is conceivable that the calcitriol therapy further increased bone resorption, in the absence of PTH, via stimulation of RANKL-mediated osteoclastogenesis. However, this seems unlikely, given the patient's low $1,25-\mathrm{OH}_{2}$ vitamin $\mathrm{D}$ level at the time of fracture. Schroth et al. [22] presented a similar case; however, changes in bone turnover markers were not reported. Fortunately, our patient responded relatively rapidly to forced saline diuresis and a single dose of pamidronate. The use of furosemide for the treatment of hypercalcemia is somewhat controversial, as it has been shown to have limited benefit and can worsen dehydration and electrolyte abnormalities if not preceded by adequate intravenous hydration [23]. In our patient, hypercalcemia, alkalosis, and renal insufficiency corrected relatively rapidly, although in some cases it may take several weeks for creatinine to normalize. When hypercalcemia is severe and prolonged, renal function may be permanently impaired.

Although rare, patients with hypoparathyroidism are at risk for developing the milk-alkali syndrome during periods of immobilization after fracture. As such, vigilant monitoring of calcium with reduction or cessation of routine calcium and vitamin D supplements may be necessary to prevent this complication.

\section{Acknowledgements}

Work by R.I. Gafni is supported by the Intramural Research Program of the NIH, NIDCR.

\section{References}

$\checkmark 1$ Albright F, Burnett CH, Cope O, Parson W: Acute atrophy of bone (osteoporosis) simulating hyperparathyroidism. J Clin Endocrinol 1941;1:711-716.

2 Rosen JF, Wolin DA, Finberg L: Immobilization hypercalcemia after single limb fractures in children and adolescents. Am J Dis Child 1978;132:560-564.

3 Sakai A, Mori T, Sakuma-Zenke M, Takeda T, Nakai K, Katae Y, Hirasawa H, Nakamura T: Osteoclast development in immobilized bone is suppressed by parathyroidectomy in mice. J Bone Miner Metab 2005;23:8-14.

4 Burkhart JM, Jowsey J: Parathyroid and thyroid hormones in the development of immobilization osteoporosis. Endocrinology 1967; 81:1053-1062.
5 Sakai A, Sakata T, Ikeda S, Uchida S, Okazaki R, Norimura T, Hori M, Nakamura T: Intermittent administration of human parathyroid hormone(1-34) prevents immobilization-related bone loss by regulating bone marrow capacity for bone cells in ddY mice. J Bone Miner Res 1999;14:1691-1699.

-6 Stewart AF, Adler M, Byers CM, Segre GV, Broadus AE: Calcium homeostasis in immobilization: an example of resorptive hypercalciuria. N Engl J Med 1982;306:1136-1140.

7 Minaire P: Immobilization osteoporosis: a review. Clin Rheumatol 1989;8(suppl 2):95103.

8 Evans RA, Bridgeman M, Hills E, Dunstan CR: Immobilisation hypercalcemia. Miner Electrolyte Metab 1984;10:244-248.
-9 Zerwekh JE, Ruml LA, Gottschalk F, Pak CY: The effects of twelve weeks of bed rest on bone histology, biochemical markers of bone turnover, and calcium homeostasis in eleven normal subjects. J Bone Miner Res 1998;13:15941601.

$\rightarrow 10$ Korkes F, Segal AB, Heilberg IP, Cattini H, Kessler C, Santili C: Immobilization and hypercalciuria in children. Pediatr Nephrol 2006;21:1157-1160.

11 Dibble JB, Penney MD: Analysis of the components of the hypercalcaemia in a 14-yearold boy following prolonged immobilisation. Acta Paediatr Scand 1983;72:207-210.

-12 Rubin MR, Dempster DW, Zhou H, Shane E, Nickolas T, Sliney J Jr, Silverberg SJ, Bilezikian JP: Dynamic and structural properties of the skeleton in hypoparathyroidism. J Bone Miner Res 2008;23:2018-2024. 
13 Sippy BW: Gastric and duodenal ulcer. JAMA 1915;64:1625-1630.

14 Felsenfeld AJ, Levine BS: Milk alkali syndrome and the dynamics of calcium homeostasis. Clin J Am Soc Nephrol 2006;1:641654.

15 Patel AM, Goldfarb S: Got calcium? Welcome to the calcium-alkali syndrome. J Am Soc Nephrol 2010;21:1440-1443.

16 Yeh BI, Sun TJ, Lee JZ, Chen HH, Huang CL: Mechanism and molecular determinant for regulation of rabbit transient receptor potential type 5 (TRPV5) channel by extracellular pH. J Biol Chem 2003;278:51044-51052.
17 Liu FY, Cogan MG: Angiotensin II: a potent regulator of acidification in the rat early proximal convoluted tubule. J Clin Invest 1987;80: 272-275.

18 Tal A, Powers K: Milk-alkali syndrome induced by $1,25(\mathrm{OH}) 2 \mathrm{D}$ in a patient with hypoparathyroidism. J Natl Med Assoc 1996;88: 313-314.

19 Altun E, Kaya B, Paydas S, Balal M: Milk alkali syndrome induced by calcitriol and calcium bicarbonate in a patient with hypoparathyroidism. Indian J Endocrinol Metab 2013; 17:S191-S193.
20 Jeong JH, Bae EH: Hypercalcemia associated with acute kidney injury and metabolic alkalosis. Electrolyte Blood Press 2010;8:92-94.

21 Boots JM, Burghouts JT, Jansen JL: Unaccountable severe hypercalcemia in a patient treated for hypoparathyroidism with dihydrotachysterol. Neth J Med 1999;54:16-20.

22 Schroth M, Dotsch J, Dorr HG: Hypercalcemia and idiopathic hypoparathyroidism. J Clin Pharm Ther 2001;26:453-455.

23 LeGrand SB, Leskuski D, Zama I: Narrative review: furosemide for hypercalcemia: an unproven yet common practice. Ann Intern Med 2008;149:259-263. 